\title{
Harmonic Analysis of Tides by Use of the Data for a Month*
}

\author{
Masamori MIYAZAKI ${ }^{* *}$
}

\section{Introduction}

In this country, the harmonic analysis of tidal observations is now carried out by two standard methods-those of G. H. DARwIN (involving the B.A. methods published between the years 1866 and 1885, and DARwIN's modifications given in 1892) and A. T. DooDson (1928). The former method is widely used when the period of analysis is a multiple of fortnight. But the computations are somewhat complicated in this method, since it requires the hourly heights to be re-written several times. In contrast with it, the latter method (usually called by the name 'T.I. method') uses some stencils, and is fairly less labourious than the former one. However, it is now applicable only when the period of analysis is about a year. Therefore, for the analysis of short period observations, DARWIN's or modified DARWIN's method is exclusively used in the present days.

The author here intends to apply the principles of the T. I. method for the short period analysis. The period of the analysis is about 30 days. Of course, the long period constituents and those of smaller amplitudes will be hardly given in our case, but important 13 constituents $\left(K_{1}, P_{1}, O_{1}, Q_{1}, K_{2}, S_{2}\right.$, $\left.L_{2}, M_{2}, N_{2}, \nu_{2}, \mu_{2}, M S_{4}, M_{4}\right)$ - which can be also computed by the DARwiN's methodwill be easily obtained in sufficient approximation.

The labour involved is surely smaller in our method than the previous ones. Two computers will sufficiently perform the total analysis of daily and monthly processes in $3-5$ hours, and the successive computations will be made within $2-3$ hours by one computor.

* Received Nov. 13, 1955.

** Kobe Marine Observatary.

\section{Notations}

The following notation are is used in this paper :

$\zeta \cdots$ the sea-level measured from a fixed datum.

$\sigma \cdots$ the speed of a constituent, in degrees $p=r$ mean solar hour.

$t \cdots$ the number of hours measured from the origin of time.

$R \cdots$ the amplitude of a constituent.

$\varepsilon \cdots$ the phase lag of a constituent at the origin of time.

$\Delta \cdots$ the constant angle proper to each constituent.

$a_{p q}, b_{p q} \cdots$ the non-dimensional numbers proper to each constituent, which are given from

$$
a_{p q}=a_{p} D_{q}, \quad b_{p q}=b_{p} D_{q} .
$$

The numerical values of $a_{p}, b_{p}$ and $D_{q}$ are already given by Doodson (loc. cit.) .

$X, e \cdots$ combinations of hourly heights.

\section{Principles of Analysis}

We first assume that the tidal variations are given by

$$
\zeta=\zeta_{0}+\Sigma R \cos (\sigma \mathrm{t}-\varepsilon),
$$

where the origin of time is chosen 336 hours after the initial time of analysis (cf. § 4). Then, the results of the monthly process will be

$$
\left.\begin{array}{l}
A_{p q}=X_{p q}+Y_{p q}=\Sigma a_{p q} R \cos \delta \\
B_{p q}=X_{p q}-Y_{p q}=\Sigma b_{p q} R \sin \delta
\end{array}\right\}
$$

where $\delta=\varepsilon-\Delta$. The numerical values of $a_{p q}$ and $b_{p q}$ are computed from Table VIIXIII of DooDson's paper.

It is not difficult to obtain $[R \cos \delta]$ or $[R \sin \delta]$ for each constituent from the algebraic equations (2), since a single constituent is roughly picked up in $A_{p q}$ and $B_{p q}$. For instance, if $p=q=2$, the absolute values of $a_{p q}$ and $b_{p q}$ for the $M_{2}$ constituent are far larger than those for other constituents. 
Therefore, the exact values of $[R \cos \delta]$ and $[R \sin \delta]$ are easily obtained by the successive approximation of only one step. 7 constituents can be separated by this method.

However, three pairs of constituents $\left(K_{1}\right.$, $P_{1} ; S_{2}, K_{0}$; and $\left.N_{2}, \nu_{2}\right)$ are hardly separated by this process because of their proximate periods. Fortunately, owing to this proximity, their mutual relations will be sufficiently given from the equilibrium theory of NewTon.

According to the equilibrium theory, we have

$$
\left.\begin{array}{l}
H\left(P_{1}\right)=0.331 H\left(K_{1}\right), \quad \kappa\left(P_{1}\right)=\kappa\left(K_{1}\right), \\
H\left(K_{2}\right)=0.272 H\left(S_{2}\right), \quad \kappa\left(K_{2}\right)=\kappa\left(S_{2}\right), \\
H\left(v_{2}\right)=0.194 H\left(N_{2}\right), \text { and } \kappa\left(v_{2}\right)=\kappa\left(N_{2}\right),
\end{array}\right\}(3)
$$

respectively. Therefore, for instance

$$
\left.\begin{array}{l}
(R \cos \delta)_{K} \\
\quad=0.272\left\{(R \cos \delta)_{S} \cos \theta-(R \sin \delta)_{s} \sin \theta\right\}, \\
(R \sin \delta)_{K} \\
\quad=0.272\left\{(R \sin \delta)_{s} \cos \theta+(R \cos \delta)_{s} \sin \theta\right\},
\end{array}\right\}
$$

where

$$
\theta=\delta_{K}-\delta_{S}=\left(v_{0}+u+\Delta\right)_{S}-\left(v_{0}+u+\Delta\right)_{K}
$$

and the suffixes $S$ and $K$ denote the variables with respect to $S_{2}$ and $K_{2}$ constituent, respecuively.

By use of the relations (4), we have

$$
\begin{aligned}
& \left.\begin{array}{l}
A_{2 K}=984.3(R \cos \delta)_{K}=\begin{array}{c}
(0.068+0.260 \cos \theta) A_{20}+0.260 \sin \theta B_{20} \\
1.068+0.520 \cos \theta
\end{array}=\beta A_{20}+\beta B_{20}, \\
B_{2 K}=-984.3(R \cos \delta)_{K}=\frac{(0.068+0.260 \cos \theta) B_{20}-0.26 \sin \theta A_{20}=\alpha B_{20}-\gamma A_{20},}{1.068+0.520 \cos \theta}
\end{array}\right\} \\
& A_{2 S}=A_{2}{ }^{0}-A_{2 \kappa}, \quad B_{2 \bar{S}}=B_{20}-B_{2 \kappa}
\end{aligned}
$$

for the first approximation. The numerical values of $\alpha, \beta$, and $\gamma$ are tabulated in Table 1 as the functions of $\theta$ (for other pairs, $\beta$ is not exactly equal to $\gamma$ ). The relations of the form (6) are to be used in the correction terms of $[R \cos \delta]$ and $[R \sin \delta]$. For the pair $\left(K_{1}, P_{1}\right)$, the separation by this method is not necessary, since the proportions of $\left(a_{1 q}\right)_{p}$ to $\left(a_{1 q}\right)_{k}$ and $\left(b_{1 q}\right)_{p}$ to $\left(b_{1 q}\right)_{k}$ are $\mathrm{ex}-$ actly constants in this case.

The final results of $H$ and $\kappa$ for these constituents are better to be given from the corrected $[R \cos \delta]$ and $[R \sin \delta]$ by use of the following formulas

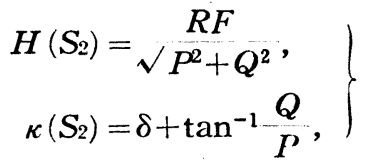

where

$$
\left.\begin{array}{l}
P=\cos \left(V_{0}+u+\Delta\right)_{s}+0.260 \cos \left(V_{0}+u+\Delta\right)_{\kappa}, \\
Q=\sin \left(V_{0}+u+\Delta\right)_{s}+0.260 \cos \left(V_{0}+u+\Delta\right)_{\kappa} .
\end{array}\right\}
$$

For the pairs $\left(K_{1}, P_{1}\right)$ and $\left(N_{2}, \nu_{2}\right)$, the coefficient 0.260 in (8) becomes 0.33 and 0.185 , respectively.

\section{Preliminary Notes}

The data are supposed to consist of hourly heights for about 30 days (Strictly speaking, the period of analysis is 713 hours). These data are usually given by the unit of centimeter. Sometimes they may be tabulated tc the tenth of a foot. In our case, the accuracy of $1 / 10$ feet is at least necessary for the analysis. At any rate, these data are to be written in the standard form of the monthly report. It is to be noted that the height at the initial time of analysis must be written in the column of $19 \mathrm{~h}$ or $20 \mathrm{th}$ column. The initial time of analysis is not necessarily adjusted to the standard time $19 \mathrm{~h}$, but matters will perhaps be simpler if we can do so.

Six stencils for the daily process $X_{1}, Y_{1}$, $X_{2}, Y_{2}, X_{4}$, and $Y_{4}$ are next to be cut to suit the form. The form of these stencils is entirely similar to that of Doodson's elementary method.

The breaks of observations will be interpolated by the following methods;

(i) For the breaks within a few hours, they will be interpolated by means of the graph of the hourly heights.

(ii) For longer breaks, they will be estimated by drawing the graphs of heights at intervals of 25 hours.

However, in our case, these breaks should 
be only ocasional and should not exceed a day at a time. If the total breaks of observations exceed two or three days, the final results will become fairly doubtful.

Day numbers are to be written from -15 to +15 . The time corresponding to the first column $(0 \mathrm{~h})$ of 16 th row (date 0 ) is taken as the origin of time. The first row (date -15) begins from the 20th column ( $19 \mathrm{~h})$, and the final row (date +15 ) ends at the 13th column (12th). Other rows consist of 24 columns.

\section{Process of analysis}

The analysis consists of 4 processes;

(i) daily process,

(ii) monthly process,

(iii) computations or $R \cos \delta$ and $R \sin \delta$ and

(iv) determination of $H$ and $\kappa$.

1. The daily process is entirely similar to that in Doodson's elementary method. Six sets of values $X_{1}, Y_{1}, X_{2}, Y_{2}, X_{4}$, and $Y_{4}$ are to be computed for the dates -14 , $-13, \cdots \cdots .+14$.

This process is better to be computed by two independent computers. A soroban is most useful for these compuations.

2. The monthly process is to be given for the following functions;

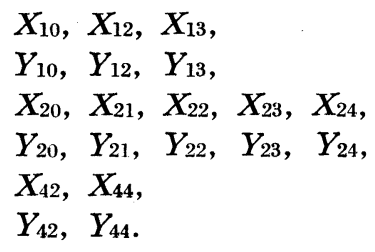

These computations are also better to be given by two indendent computers,

If we further compute the functions of the form $X_{2 a}, Y_{2 a}$ in addition to these functions, the check of the results will be easily made. However, as long as the computations are carried out very carefully and checked by two computers, further check by this method will be no longer necessary.

3. We must then prepare the forms of Table 6 and Table 7. Compute the numerical values of $V_{0}+u$ and $F$ at the origin of time for each constituent, and enter the values of $\Delta$ from Table 2 .

4. Compute $\theta$ for two pairs $\left(S_{2}, K_{2}\right)$ and $\left(N_{2}, \nu_{2}\right)$, and then compute the values of $\alpha, \beta$, and $\gamma$ from Table 1 . Then, it is very easy to estimate $A_{2 \kappa}, B_{2 K}, A_{2 N}, B_{2 N}, A_{2 \nu}$, and $B_{2 \nu}$ by use of the values $A_{20}, B_{20}, A_{23}$, and $B_{23}$ (cf. formulas (6)).

5. Prepare the tables for the computations of $R \cos \delta$ and $R \sin \delta$. The form is as follows.

\begin{tabular}{|c|c|c|c|c|c|c|c|c|}
\hline \multicolumn{2}{|c|}{ Principal term } & \multicolumn{3}{|c|}{ Correction terms } & \multirow{2}{*}{ Sum } & \multirow{2}{*}{ Divisor } & \multirow{2}{*}{$R \cos \delta$} & \multirow{2}{*}{ Constituent } \\
\hline Symbol & Value & $A_{10}$ & $A_{12}$ & $A_{13}$ & & & & \\
\hline$A_{10}$ & & & & & & 859.2 & & $K_{1}^{*}$ \\
\hline$\cdots \cdots$ & & & & & & $\cdots \cdots$ & & \\
\hline
\end{tabular}

Enter the numerical values of $A_{p q}, B_{p q}$ in them, and compute the correction terms by multiplying the tabular values (Tables 3 and 4). The numerical values of $R \cos \delta$ and $R \sin \delta$ are finally computed in these tables.

6a. $H$ and $\kappa$ for 7 constituents $\left(Q_{1}, O_{1}\right.$, $L_{2}, M_{2}, \mu_{2}, M S_{4}$, and $M_{4}$ ) are computed by use of Table 5 . Entering the numerical values of $R \cos \delta$ and $R \sin \delta$ in this table, we can easily compute the final results.

$6 \mathrm{~b}$. For other 6 constituents, the numerical values of $H$ and $\kappa$ are given from formulas (7) and the similar formulas. We shall make use of Table 6 for these computations.

7. Lastly, all computations are to be checked once more. 
6. Examples of analysis and the comparison with the results given by DARWIN's method

For an example, we shall tabulate the results of analysis for the Akashi Harbour (Lat. $34^{\circ} 39^{\prime} \mathrm{N}$, Long. $135^{\circ} 00^{\prime} \mathrm{E}$ ) in Table 7 . These results are obtained from the continuous records measured by the tide gauge of Richard type in August, 1955.

For the purpose of comparison, we also show the results given by DARwin's method in this table. These two results are in good agreement with each other except for the constituents of smaller amplitudes.

Acknowledgements-This investigation has been carried out by the aid of the ' $\mathrm{Re}$ search Committee of the Akashi Straight,' established in the Kobe City. The author here wishes to express his best thanks for Dr. C. HaRAGUchi, Mayor of the Kobe City, Dr. Y. Matsudaira, Director of the Kobe Marine Observatory, Dr. M. NaKano, Chief of the Oceanographical Laboratory, Meteorological Research Institute, Tokyo, and other members of the Committee, who kindly guide him and gave him the chance of the investigation. The author also wishes to express his best thanks to Miss K. Morimoto for her aid in the preparation of this paper.

\section{References}

DARWIN, G. H., 1907 :

"Scientific Papar, Part 1 (Oceanic tides)", Art 1-7, p. 1-327, Cambridge.

Doodson, A. T., 1928 :

"The analysis of tidal observations." Phil.

Trans. Roy. Soc. London, A 227, p. 223-279.
Table 1. Numerical values of $\alpha, \beta$, and $\gamma$ against for $\theta$ 's values at the intervals of 10 . (This table is of course used for the determination of $\alpha, \beta$, and $\gamma$ at the intermediate values $\theta$. However, it is better to plot these these values on diagrams, and to make use these diagrams for this purpose.

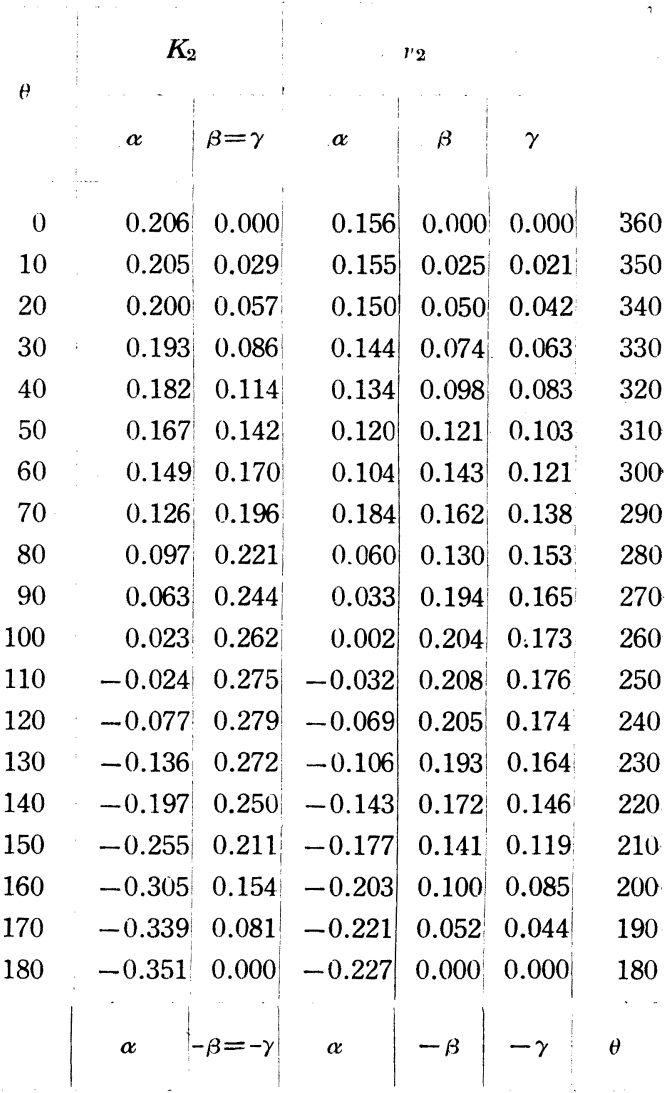

Table 2. Values of $A$

\begin{tabular}{cc|cc|c|c}
\hline$Q_{1}$ & 207.68 & $N_{2}$ & 80.82 & $K_{2}$ & 106.27 \\
\hline$O_{1}$ & 216.12 & $\iota_{2}$ & 81.94 & $M_{4}$ & $88 . g 1$ \\
\hline$P_{1}$ & 231.86 & $M_{2}$ & 89.25 & $M S_{4} 104.25$ \\
\hline$K_{1}$ & 233.14 & $L_{2}$ & 97.69 & \\
\hline$\mu_{2}$ & 73.51 & $S_{2}$ & 105.00 & \\
\hline
\end{tabular}


Table 3. Computation of $R \cos \delta$

\begin{tabular}{|c|c|c|c|c|c|c|c|c|}
\hline \multirow{2}{*}{$\begin{array}{l}\text { Principal } \\
\text { function }\end{array}$} & \multicolumn{6}{|c|}{ Correction terms: multiples of } & \multirow{2}{*}{ Divisor } & \multirow{2}{*}{ Constituent } \\
\hline & $A_{10}$ & $A_{12}$ & $A_{13}$ & & & & & \\
\hline$A_{10}$ & - & -0.104 & 0.029 & & & & 852.2 & $\mathrm{~K}_{1} *$ \\
\hline$A_{12}$ & 0.004 & - & 0.206 & & & & 1062.9 & $O_{1}$ \\
\hline \multirow[t]{3}{*}{$A_{13}$} & -0.002 & -0.074 & - & & & & 1062.8 & $Q_{1}$ \\
\hline & \multicolumn{6}{|c|}{ Correction terms: multiples of } & & \\
\hline & $A_{2 K}$ & $A_{12}$ & $A_{22}$ & $A_{2 N}$ & $A_{2 \nu}$ & $A_{24}$ & & \\
\hline$A_{20}$ & - & -0.104 & 0.017 & 0.006 & 0.044 & 0.019 & 984.3 & $S_{2}^{*}$ \\
\hline$A_{21}$ & -0.049 & - & -0.004 & -0.054 & 0.071 & -0.092 & 928.3 & $L_{2}$ \\
\hline$A_{22}$ & 0.012 & 0.030 & - & 0.124 & -0.088 & 0.096 & 1099.0 & $M_{2}$ \\
\hline$A_{23}$ & -0.007 & -0.068 & -0.018 & - & -- & -0.013 & 1084.3 & $N_{2}^{*}$ \\
\hline \multirow[t]{3}{*}{$A_{24}$} & 0.005 & 0.048 & 0.016 & -0.037 & 0.083 & - & 1103.5 & $\mu_{2}$ \\
\hline & \multicolumn{6}{|c|}{ Correction terms: multiples of } & & \\
\hline & $A_{42}$ & $A_{44}$ & & & & & & \\
\hline$A_{42}$ & - & 0.088 & & & & & 509.8 & $M S_{4}$ \\
\hline$A_{44}$ & 0.016 & - & & & & & 503.8 & $M_{i}$ \\
\hline
\end{tabular}

Table 4. Computaticn of $R \sin \delta$

\begin{tabular}{|c|c|c|c|c|c|c|c|c|}
\hline \multirow{2}{*}{$\begin{array}{l}\text { Principal } \\
\text { functicn }\end{array}$} & \multicolumn{6}{|c|}{ Correction terms: multiples of } & \multirow{2}{*}{ Divisor } & \multirow{2}{*}{ Constituent } \\
\hline & $B_{10}$ & $B_{12}$ & $B_{13}$ & & & & & \\
\hline$B_{10}$ & - & -0.020 & 0.029 & & & & -661.0 & $K_{1}^{*}$ \\
\hline$B_{12}$ & 0.004 & - & 0.206 & & & & -703.4 & $O_{1}$ \\
\hline \multirow[t]{3}{*}{$B_{13}$} & -0.002 & -0.074 & - & & & & -740.3 & $Q_{1}$ \\
\hline & \multicolumn{6}{|c|}{ Correction terms: multiples of } & & \\
\hline & $B_{2 K}$ & $B_{21}$ & $B_{22}$ & $B_{2 N}$ & $B_{2 \nu}$ & $B_{\mathbf{2 4}}$ & & \\
\hline$B_{20}$ & - & -0.104 & 0.017 & -0.005 & 0.041 & 0.018 & -984.3 & $S_{2}^{*}$ \\
\hline$B_{21}$ & 0.049 & - & -0.004 & 0.049 & -0.065 & -0.085 & -905.6 & $L_{2}$ \\
\hline$B_{22}$ & -0.012 & 0.031 & - & -0.114 & 0.082 & 0.088 & -1042.1 & $M_{2}$ \\
\hline$B_{23}$ & 0.007 & -0.068 & -0.018 & - & - & -0.122 & -9992 & $\mathrm{~N}_{2}^{*}$ \\
\hline \multirow[t]{3}{*}{$B_{54}$} & -0.005 & 0.048 & 0.016 & 0.034 & -0.077 & - & -991.9 & $\mu_{2}$ \\
\hline & \multicolumn{6}{|c|}{ Correction terms: multiples cf } & & \\
\hline & $B_{42}$ & $B_{44}$ & & & & & & \\
\hline$B_{42}$ & - & \multirow{2}{*}{\multicolumn{2}{|c|}{0.088}} & & & & -847.8 & $M S_{4}$ \\
\hline$B_{44}$ & 0.016 & & & & & & -805.1 & $M_{5}$ \\
\hline
\end{tabular}


Table 5. Example of computation of $H$ and $\kappa$

(Akashi, August, 1955)

\begin{tabular}{|c|c|c|c|c|c|c|c|}
\hline & $O_{1}$ & $Q_{1}$ & $L_{2}$ & $\boldsymbol{M}_{2}$ & $\mu_{2}$ & $M S_{4}$ & $M_{4}$ \\
\hline$R \cos \delta$ & -12.57 & -2.23 & 0.80 & 8.21 & -0.68 & -1.27 & 1.75 \\
\hline$R \sin \delta$ & 16.27 & 2.90 & 0.63 & 11.22 & -3.44 & 0.43 & 0.81 \\
\hline$R$ & 20.56 & 3.66 & 1.02 & 13.90 & 3.51 & 1.34 & 1.93 \\
\hline$F$ & 0.998 & 0.998 & 0.797 & 0.886 & 0.996 & 0.996 & 0.991 \\
\hline$H=R F$ & 20.51 & 3.65 & 0.81 & 13.84 & 3.39 & 1.33 & 1.91 \\
\hline$\delta$ & 127.68 & 127.58 & 38.25 & 53.80 & 258.83 & 161.46 & 24.95 \\
\hline$V_{0}+u$ & 206.62 & 186.64 & 289.47 & 91.88 & 181.56 & 91.88 & 183.66 \\
\hline$\Delta$ & 216.12 & 207.68 & 97.69 & 89.25 & 73.51 & 104.25 & 88.51 \\
\hline$\kappa=\mathrm{sum}$ & 190.42 & 161.90 & 65.51 & 234.93 & 153.90 & 357.53 & 297.12 \\
\hline
\end{tabular}

Table 6. Exauple of computaticn of $H$ and $\kappa$ (ii)

Sheet 1 .

(Akashi, August, 1955)

\begin{tabular}{l|r|r|r|r|r|r}
\hline & $K_{1}$ & $P_{1}$ & \multicolumn{1}{c}{$S_{2}$} & \multicolumn{1}{c}{$K_{2}$} & \multicolumn{1}{c}{$N_{2}$} & $I^{\prime 2}$ \\
\hline$V_{0}+u$ & 241.06 & 127.74 & 0.00 & 302.41 & 71.79 & 201.55 \\
$\Delta$ & 233.14 & 231.86 & 105.00 & 106.27 & 80.82 & 81.94 \\
$\eta=$ sum & 114.20 & 359.60 & 105.00 & 46.68 & 152.61 & 283.49 \\
\hline $\cos \eta$ & -0.4099 & 1.0000 & -0.2588 & 0.6602 & -0.8879 & 0.2332 \\
$\sin \eta$ & 0.9121 & -0.0070 & 0.9659 & 0.7511 & 0.4599 & -0.9724 \\
\hline
\end{tabular}

Sheet 2.

\begin{tabular}{|c|c|c|c|}
\hline & $K_{1}^{*}$ & $S_{2}^{*}$ & $\mathrm{~N}_{2}^{*}$ \\
\hline$\theta$ & - & 56.32 & -130.88 \\
\hline$P$ & -0.0679 & -0.0871 & - 0.8448 \\
\hline $\boldsymbol{Q}$ & 0.9098 & 1.1612 & 0.2800 \\
\hline$R \cos \delta$ & -10.68 & -8.22 & 1.84 \\
\hline$R \sin \delta$ & 21.77 & 6.73 & 3.64 \\
\hline$R$ & 24.24 & 10.62 & 4.08 \\
\hline$F$ & 0.998 & 1.000 & 0.996 \\
\hline$H=\frac{R E}{\sqrt{ } P^{2} Q^{2}}$ & 26.52 & 9.12 & 4.56 \\
\hline$\delta$ & 116.13 & 140.68 & 116.82 \\
\hline $\tan ^{-1} Q / F$ & 94.27 & 84.28 & 161.67 \\
\hline$\kappa=\operatorname{sum}$ & 210.40 & 234.96 & 278.46 \\
\hline$n$ & 0.331 & 0.272 & 0.194 \\
\hline$n H$ & 8.78 & 2.48 & 0.88 \\
\hline
\end{tabular}

Table 7. Harmcnic constants of tides at the Akashi Harbour

\begin{tabular}{|c|c|c|c|c|}
\hline \multirow{2}{*}{ Constituent } & \multicolumn{2}{|c|}{$\begin{array}{c}\text { Method in this } \\
\text { paper }\end{array}$} & \multicolumn{2}{|c|}{$\begin{array}{l}\text { Darwin's } \\
\text { method }\end{array}$} \\
\hline & $H$ & $\kappa$ & $H$ & $\kappa$ \\
\hline$K_{1}$ & 26.52 & 210.40 & 25.62 & 211.57 \\
\hline$O_{1}$ & 20.51 & 190.42 & 20.54 & 189.99 \\
\hline$P_{1}$ & 8.78 & 210.40 & 8.54 & 211.57 \\
\hline$Q_{1}$ & 3.65 & 161.90 & 3.61 & 171.95 \\
\hline$M_{2}$ & 13.84 & 234.93 & 14.71 & 240.15 \\
\hline$S_{2}$ & 9.12 & 234.96 & 9.53 & 233.79 \\
\hline$K_{2}$ & 2.48 & 234.96 & 2.60 & 233.79 \\
\hline$N_{2}$ & 4.56 & 278.49 & 3.13 & 237.26 \\
\hline$L_{2}$ & 0.81 & 65.51 & 0.61 & 177.47 \\
\hline$\nu_{2}$ & 0.88 & 278.49 & 0.61 & 237.26 \\
\hline$\mu_{2}$ & 3.49 & 153.90 & 4.48 & 152.44 \\
\hline$M_{4}$ & 1.91 & 297.12 & 1.49 & 334.51 \\
\hline$M S_{4}$ & 1.33 & 357.53 & 1.26 & 333.54 \\
\hline
\end{tabular}

